\title{
Glicerina bruta na dieta de bovinos de corte confinados: efeito sobre o hemograma
}

\author{
Crude glycerin on feedlot beef cattle diets: effect on hemogram
}

\author{
David Attuy Vey da SILVA ${ }^{1}$; Eric Haydt Castello Branco VAN CLEEF ${ }^{1}$; Jane Maria Bertocco \\ EZEQUIEL $^{1}$; André Pastori D 'ÁUREA ${ }^{1}$;Vanessa Ruíz FÁVARO ${ }^{1}$
}

${ }^{1}$ Faculdade de Ciências Agrárias e Veterinárias da Universidade Estadual Paulista , Jaboticabal-SP, Brasil

\begin{abstract}
Resumo
Objetivou-se com este estudo avaliar o hemograma de bovinos alimentados com glicerina bruta. Trinta novilhos da raça Nelore foram utilizados para as colheitas de sangue, as quais foram realizadas a cada 28 dias. Os animais foram mantidos confinados durante 103 dias e foram alimentados com cinco dietas contendo $0,7,5,15,22,5$ e $30 \%$ de glicerina bruta com base na matéria seca, formuladas na proporção volumoso:concentrado de 30:70 sendo a silagem de milho o volumoso e o concentrado composto por milho em grão, casca de soja, farelo de girassol, glicerina, calcário calcítico, fostatobicálcico e sal comum. O delineamento utilizado foi em blocos casualisados com cinco tratamentos. Contrastes ortogonais foram usados para determinar os efeitos linear, quadrático, cúbico e tratamento com glicerina $\times$ controle. Não houve efeito dos tratamentos $(\mathrm{P}>0,05)$ sobre as concentrações sanguíneas de hemácias, hematócrito, basófilos, eosinófilos, neutrófilos bastonetes e linfócitos, porém houve efeito linear sobre as concentrações de plaquetas, hemoglobina e monócitos $(\mathrm{P}=0,009, \mathrm{P}=0,001$ e $\mathrm{P}=0,043$, respectivamente), e efeito cúbico sobre as concentrações de leucócitos totais e neutrófilos segmentados $(\mathrm{P}=0,008$ e $\mathrm{P}=0,004$, respectivamente). Dietas para bovinos de corte, contendo relação volumoso:concentrado 30:70 aditivadas com glicerina bruta, promovem alterações no eritrograma e no leucograma destes animais.
\end{abstract}

Palavras-chave: Confinamento. Coprodutos. Sangue. Ruminantes.

\begin{abstract}
The aim of this study was to evaluate the hemogram in cattle fed crude glycerin. 30 Nellore steers, were used for blood sampling, which were done each 28 days. The animals were confined for 103 days and were fed with five diets containing $0,7.5,15,22.5$ and $30 \%$ crude glycerin based on dry matter, formulated in a forage:concentrate ratio of 30:70. Corn silage was used as forage and the concentrate was composed by corn grain, soybean hulls, sunflower meal, glycerin, limestone, dicalcium phosphate and salt. The experimental design was a randomized block with five treatments. Orthogonal contrasts were used to determine the linear, quadratic, cubic effects and gliceryn treatments $\times$ the control one. There was no treatment effect $(\mathrm{P}>0,05)$ on blood concentrations of erythrocytes, hematocrit, basophils, eosinophils, band neutrophils and lymphocytes, but were linearly effect on the concentrations of platelets, hemoglobin and monocytes $(\mathrm{P}=0.009, \mathrm{P}=0.001$ and $\mathrm{P}=0.043$, respectivelly), and cubic effect on the concentrations of total leukocytes and segmented neutrophils $(\mathrm{P}=0.008$ and $\mathrm{P}=0.004$, respectivelly). Diets for beef cattle, with forage:concentrate ratio of 30:70 added with crude glycerin promote changes in the erytrhogram and leucogram of these animals.
\end{abstract}

Keywords: Blood. Co-products. Feedlot. Ruminants.

\section{Introdução}

O sistema brasileiro de produção de carne bovina baseia-se em pastagens, porém, o confinamento apresenta diversas vantagens, como a redução na idade de abate do animal, produção de carne de melhor qualidade, aumento na taxa de desfrute reduzindo a ociosidade dos frigoríficos na entressafra, maior giro de capital, melhor aproveitamento das áreas de pastagens para outras categorias animais e elevada produção de adubo orgânico ${ }^{1}$.
A produção em escala comercial de culturas voltadas à produção de biodiesel vem aumentando e acar-

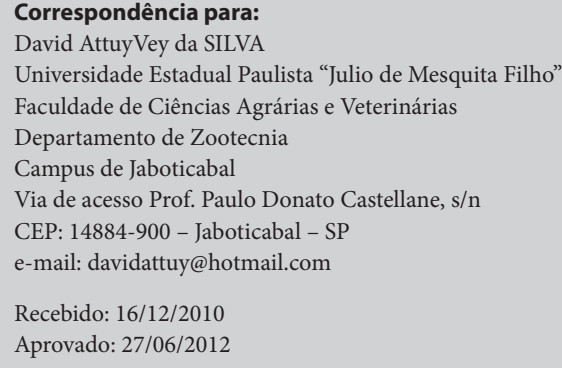


reta a geração de produtos que necessitam de destino econômico e ecologicamente correto. Os produtos gerados na cadeia produtiva do biodiesel devem ser focos de análises mais detalhadas, pois podem ser um fator determinante para a viabilidade econômica da produção desse combustível. Entre os principais pode-se citar a glicerina. Entretanto, existem poucos estudos acerca do aproveitamento desse produto como elemento de viabilização da cadeia produtiva.

Desde $1^{\circ}$ de janeiro de 2010, o óleo diesel comercializado em todo o Brasil contém 5\% de biodiese ${ }^{2}$. Com a entrada do B5 no mercado Brasileiro, temos um excedente de $150 \mathrm{mil}$ ton/ano de glicerina, extrapolando em muito o consumo de glicerina no mercado nacional. Assim, para a viabilização econômica do biodiesel, deve-se encontrar um fim benéfico para a glicerina excedente gerada. Uma alternativa é o uso da glicerina na alimentação de animais, porém os resíduos de metanol e outros contaminantes podem ser um problema, gerando alterações metabólicas nos animais acarretando em prejuízos aos animais e aos produtores.

A glicerina é um produto de valor comercial e que possui inúmeras aplicações industriais, porém a glicerina obtida no processo de produção do biodiesel apresenta-se mais escura e contendo algumas impurezas. Sendo assim, há um grande interesse na sua purificação e no seu reaproveitamento, pois isto levaria à viabilização do processo, permitindo que o biodiesel se torne competitivo no mercado de combustíveis ${ }^{3}$.A principal rota de obtenção do biodiesel é a partir da transesterificação de óleos vegetais com álcoois (metanol e etanol), usando catálise básica. Do ponto de vista químico, o óleo vegetal usado na produção de biodiesel é um triglicerídeo, ou seja, um triéster derivado da glicerina. Sob ação de um catalisador básico e na presença de metanol ou etanol, o óleo sofre uma transesterificação formando três moléculas de ésteres metílicos ou etílicos dos ácidos graxos, que constituem o biodiesel em sua essência, liberando uma molécula de glicerol ou glicerina ${ }^{4}$.

Os parâmetros sanguíneos têm sido utilizados mundialmente para avaliar o estado de saúde dos animais e também como indicadores de estresse ${ }^{5}$. Vários fatores como espécie, sexo, idade, estado fisiológico, hora do dia, umidade relativa do ar, temperatura ambiente, atividade muscular e o estado nutricional de cada animal podem provocar alterações desses parâmetros. Alguns pesquisadores já demonstraram o interesse em estudar a influência de alguns destes fatores sobre o hemograma de bovinos, tendo inclusive, estabelecido alguns padrões hematológicos ${ }^{6,7,8}$.

Desequilíbrios entre os nutrientes que ingressam no organismo animal, sua biotransformação e a eliminação das substâncias resultantes podem ocasionar alterações no metabolismo animal. A consequência direta dessa condição é a ocorrência das doenças metabólicas, também conhecidas como doenças da produção. Muitos desses desequilíbrios podem provocar doenças subclínicas que são de difícil percepção, limitando a produção de um modo persistente, provocando diminuição da produção e ocasionando perdas na rentabilidade do produtor.

A composição bioquímica do sangue reflete de modo fiel a situação metabólica dos tecidos animais, de forma a poder avaliar lesões teciduais, transtornos no funcionamento de órgãos, adaptação do animal diante de desafios nutricionais e fisiológicos e desequilíbrios metabólicos específicos ou de origem nutricional ${ }^{9}$. A ocorrência de distúrbios metabólicos é muito mais comum em confinamentos que utilizam rações com elevado teor de grãos de cereais e estes distúrbios podem ser detectados pelo estudo dos perfis sanguíneos ${ }^{10}$. Portanto, o objetivo deste estudo foi avaliar o hemograma de bovinos de corte recebendo glicerina bruta. 


\section{Material e Método}

O experimento foi conduzido nas dependências da Unidade Animal de Estudos Digestivos e Metabólicos pertencente ao Departamento de Zootecnia da Faculdade de Ciências Agrárias e Veterinárias da Universidade Estadual Paulista "Julio de Mesquita Filho", Campus de Jaboticabal, São Paulo.

Durante o período pré-experimental, os animais foram identificados individualmente com brincos e everminados. Foram alojados em baias individuais de $16 \mathrm{~m}^{2}$, com piso concretado e parcialmente cobertas, providas de bebedouro e cocho. Durante os períodos pré-experimental e experimental as baias e bebedouros foram limpos semanalmente.

Trinta novilhos da raça Nelore, com aproximadamente 18 meses de idade e $277,70 \pm 23,8 \mathrm{~kg}$ de peso vivo médio foram utilizados para colheitas de sangue durante pesagens do ensaio de produção. O período total de confinamento foi de 103 dias, sendo os primeiros 21 dias destinados à adaptação dos animais ao manejo, às instalações e às dietas. A alimentação foi oferecida duas vezes ao dia, às $8 \mathrm{~h}$ e às $17 \mathrm{~h}$. $\mathrm{O}$ concentrado e o volumoso foram pesados separadamente e fornecidos em quantidades iguais em cada hora do fornecimento. A oferta dos alimentos foi ajustada diariamente para sobra de aproximadamente $10 \%$, aferidos anteriormente à refeição da manhã.

Os animais foram submetidos à colheita de sangue através de punção da veia coccígea com os animais contidos em tronco, após os 21 dias de adaptação, após quatro e oito semanas da adaptação e no dia anterior ao abate dos animais, totalizando quatro colheitas no período de 103 dias de confinamento. O delineamento utilizado foi em blocos casualisados com seis repetições por tratamento. Os contrastes ortogonais foram utilizados para verificar os efeitos linear, quadrático, cúbico e o efeito dos tratamentos versus o tratamento controle, da inclusão de glicerina nas dietas através do programa estatístico SAS (SAS, 2001). As amostras foram analisadas no Laboratório do Departamento de Clínica e Cirurgia Veterinária da FCAV/Unesp Campus de Jaboticabal quanto às concentrações sanguíneas dos valores eritrocitários (hemácias, hemoglobina e hematócrito), leucocitários (leucócitos totais, neutrófilos bastonetes e segmentados, eosinófilos, basófilos, monócitos e linfócitos) e contagem de plaquetas.

As dietas experimentais foram formuladas para atender as exigências mínimas de nutrientes, de acordo com o NRC (1996). Cinco dietas isoprotéicas e isoenergéticas foram formuladas na proporção volumoso:concentrado de 30:70 sendo a silagem de milho o volumoso e o concentrado composto por milho em grão, casca de soja, farelo de girassol, glicerina, calcário calcítico, fosfato bicálcico e sal comum. As dietas experimentais foram: GLI 0 - controle, sem adição de glicerina; GLI 7,5 - 7,5\% de glicerina na dieta; GLI 15 - 15\% de glicerina na dieta; GLI 22,5 - 22,5\% de glicerina na dieta; GLI 30 - 30\% de glicerina na dieta. Na tabela 1 podem ser visualizadas as composições percentual e bromatológica das dietas experimentais.

\section{Resultados e Discussão}

No quadro eritrocitário somente houve efeito linear decrescente $(\mathrm{P}=0,001)$ dos tratamentos sobre a concentração de hemoglobina. Não houve efeito dos tratamentos $(\mathrm{P}>0,05)$ sobre as concentrações de hemácias e hematócrito. Foi observado efeito linear crescente $(\mathrm{P}=0,009)$ dos tratamentos sobre a contagem de plaquetas. Os valores médios encontrados para a contagem global de hemácias e plaquetas, teor de hemoglobina e determinação do hematócrito estão dentro dos valores sugeridos como normais pela literatura $^{11,12,13}$ e são mostrados na tabela 2.

$\mathrm{Na}$ prática, a contagem global de hemácias, determinação do hematócrito e da concentração de hemoglobina, são úteis na avaliação da anemia, que é caracterizada por uma redução destes parâmetros. Pode-se 
Tabela 1 - Porcentagem dos ingredientes e estimativa da composição bromatológica dos tratamentos experimentais (\% MS)

\begin{tabular}{|c|c|c|c|c|c|}
\hline \multirow{2}{*}{ Ingredientes } & \multicolumn{5}{|c|}{ Tratamentos } \\
\hline & GLI $0^{1}$ & GLI $7,5^{2}$ & GLI $15^{3}$ & GLI $22,5^{4}$ & GLI $30^{5}$ \\
\hline Silagem de milho & 30,00 & 30,00 & 30,00 & 30,00 & 30,00 \\
\hline Milho & 35,00 & 25,50 & 18,00 & 12,50 & 5,00 \\
\hline Casca de soja & 19,20 & 18,05 & 14,55 & 8,90 & 5,45 \\
\hline Farelo de girassol & 14,60 & 17,80 & 21,30 & 24,90 & 28,40 \\
\hline Glicerina & 0,00 & 7,50 & 15,00 & 22,50 & 30,00 \\
\hline Sal comum & 0,50 & 0,50 & 0,50 & 0,50 & 0,50 \\
\hline Calcário calcítico & 0,70 & 0,65 & 0,55 & 0,70 & 0,65 \\
\hline Fosfato bicálcico & 0,00 & 0,10 & 0,10 & 0,00 & 0,00 \\
\hline \multirow[t]{2}{*}{ Total } & 100,00 & 100,00 & 100,00 & 100,00 & 100,00 \\
\hline & \multicolumn{5}{|c|}{ Composição Bromatológica } \\
\hline PB (\% MS) & 12,20 & 12,21 & 12,22 & 12,20 & 12,21 \\
\hline EM (Mcal/kg MS) & 2,53 & 2,52 & 2,52 & 2,53 & 2,52 \\
\hline $\mathrm{EE}(\%)$ & 2,90 & 2,58 & 2,31 & 2,09 & 1,81 \\
\hline $\mathrm{Ca}(\%)$ & 0,56 & 0,56 & 0,55 & 0,57 & 0,55 \\
\hline P (\%) & 0,32 & 0,32 & 0,35 & 0,34 & 0,34 \\
\hline
\end{tabular}

${ }^{1}$ Tratamento controle sem adição de glicerina; ${ }^{2}$ Adição de $7,5 \%$ de glicerina na MS da dieta; ${ }^{3}$ Adição de $15 \%$ de glicerina na MS da dieta; ${ }^{4}$ Adição de $22,5 \%$ de glicerina na MS da dieta; ${ }^{5}$ Adição de 30,0\% de glicerina na MS

Tabela 2 - Concentrações de hemácias, hemoglobina, hematócrito e plaquetas

\begin{tabular}{|c|c|c|c|c|c|c|c|c|c|c|c|}
\hline \multirow{2}{*}{ Ítens } & \multicolumn{5}{|c|}{ Glicerina bruta, (\%) } & \multirow{2}{*}{ Média } & \multicolumn{5}{|c|}{ Contrastes } \\
\hline & 0 & 7,5 & 15 & 22,5 & 30 & & $\mathrm{~L}^{\mathrm{a}}$ & $Q^{b}$ & $\mathrm{C}^{c}$ & 0 vs.gli ${ }^{\mathrm{d}}$ & $\mathrm{CV}(\%)^{\mathrm{e}}$ \\
\hline $\mathrm{HE}^{\mathrm{f}}$ & 8,54 & 8,27 & 8,38 & 8,34 & 8,24 & 8,35 & NS & NS & NS & NS & 15,5 \\
\hline $\mathrm{HG}^{\mathrm{g}}$ & 12,87 & 12,03 & 12,50 & 12,02 & 10,92 & 12,06 & ** & NS & NS & * & 14,7 \\
\hline $\mathrm{HT}^{\mathrm{h}}$ & 39,10 & 34,50 & 36,61 & 36,83 & 36,95 & 36,74 & NS & NS & NS & NS & 19,3 \\
\hline PLA $^{\mathrm{i}}$ & 203,8 & 257,5 & 278,8 & 309,4 & 294,5 & 270,3 & $* *$ & NS & NS & * & 48,6 \\
\hline
\end{tabular}

${ }^{\mathrm{a}}$ Linear; ${ }^{\mathrm{b} Q u a d r a ́ t i c o ; ~}{ }^{\mathrm{C} C u ́ b i c o}$; ${ }_{\mathrm{d}}^{\mathrm{d}}$ ontrole versus os outros tratamentos; ${ }^{\mathrm{C} C o e f i c i e n t e ~ d e ~ v a r i a c ̧ a ̃ o, ~}{ }^{\mathrm{f}} \mathrm{Hemácias}\left(\mathrm{x} 10^{6} / \mu \mathrm{L}\right),{ }^{\mathrm{g}} \mathrm{Hemoglo}-$ bina $(\mathrm{g} / \mathrm{dL}),{ }^{\text {h}}$ Hematócrito $(\%),{ }^{\text {PPlaquetas }}\left(\mathrm{x} 10^{3} / \mu \mathrm{L}\right) ;{ }^{*}<0,05 ;{ }^{* *}<0,01$; NS = Não significativo

assumir então, que os animais não apresentaram o quadro de anemia, pois não foi observada a redução do hematócrito, número de eritrócitos ou da concentração de hemoglobina dos animais em nenhum dos tratamentos experimentais, quando comparados aos valores sugeridos como normais pela literatura ${ }^{14,15,16}$.

Devido ao efeito linear decrescente observado sobre a concentração de hemoglobina perante os tratamentos, pode-se inferir que o aumento na concentração de glicerina pode causar efeitos hematológicos que predispõem à anemia, porém apenas com esse parâmetro isolado não é possível a identificação de alterações significativas no metabolismo animal. Possivelmente, com um maior período de ação da glicerina no organismo animal, outros parâmetros como, contagem de hemácias e concentração de hematócrito, poderiam ser alterados. 
A trombocitose pode ocorrer devido à proliferação neoplásica na medula óssea, estimulação da medula óssea ou pela contração esplênica, que aumenta o número de plaquetas na circulação sanguínea. Apesar de ter sido evidenciado aumento no número de plaquetas com o aumento da concentração de glicerina, não houve trombocitose, pois os valores não ultrapassaram os limites sugeridos como normais pela literatura ${ }^{17}$. Sendo assim, o aumento das plaquetas pode ter sido devido às alterações metabólicas causadas pela alta ingestão de concentrado associado à glicerina, podendo ter ocorrido maior atividade medular e/ou esplênica.

Lee, Roussel e Beatty ${ }^{14}$, ao estudarem o efeito de estresse em bovinos, observaram que os animais apresentaram alterações hematológicas no quadro eritrocitário quando submetidos ao estresse. Devido às principais respostas ao estresse serem medidas pela adrenalina e cortisol pode-se verificar mudança no quadro hematológico minutos após a secreção de adrenalina enquanto as alterações decorrentes da ação dos corticosteroides só se manifestam poucas horas após o estresse sofrido. A adrenalina causa esplenocontração com consequente liberação de uma massa de eritrócitos para a circulação sanguínea. Este efeito ocorre três minutos após uma descarga de adrenalina, sendo que o aumento no número de eritrócitos pode ser superior a $38 \%$ em ovinos ${ }^{19}$.

A contração esplênica, como causa de eritrocitose relativa, ocorre principalmente em indivíduos excitáveis $^{20}$, embora essa resposta possa ocorrer em todas as espécies ${ }^{21}$. Portanto, como os animais foram submetidos às colheitas de sangue em tronco de contenção, esse manejo não gerou estresse suficiente para alterar o quadro eritrocitário dos animais. A alta conversão alimentar imposta aos animais confinados recebendo dietas com alto teor de concentrado pode ter desencadeado alterações metabólicas com consequentes alterações hematológicas.
Foi observado efeito cúbico $(\mathrm{P}=0,008$ e $\mathrm{P}=0,004)$ dos tratamentos sobre as concentrações de leucócitos totais e neutrófilos segmentados, respectivamente e efeito linear crescente $(\mathrm{P}=0,043)$ sobre a concentração de monócitos. A maior média encontrada para contagem de leucócitos totais e neutrófilos segmentados foi observada no tratamento com adição de $22,5 \%$ de glicerina e a menor média foi observada no tratamento com adição de $30 \%$ e $7,5 \%$ de glicerina, respectivamente.

Não houve efeito dos tratamentos $(\mathrm{P}>0,05)$ sobre as concentrações sanguíneas de basófilos, eosinófilos, neutrófilos bastonetes e linfócitos. Os valores encontrados para leucócitos totais se mostraram acima dos sugeridos como normais por Kaneko, Harvey e Bruss $^{11}$ e Aengwanich, Chantiratikul e Pamok ${ }^{12}$, porém dentro dos valores encontrados por Oliver et al. ${ }^{13} \mathrm{e}$ Birgel Junior et al. ${ }^{18}$. Os valores encontrados para neutrófilos bastonetes e monócitos estão dentro dos sugeridos como normais por Kaneko, Harvey e Bruss ${ }^{11}$, Aengwanich, Chantiratikul e Pamok ${ }^{12}$, Oliver et al. ${ }^{13} \mathrm{e}$ Birgel Junior et al. ${ }^{18}$.

Entretanto, ocorreu aumento dos neutrófilos segmentados quando comparados aos valores sugeridos como normais por Aengwanich, Chantiratikul e Pamok ${ }^{12}$ e Oliver et al. ${ }^{13}$, mas se encontram dentro dos intervalos sugeridos como normais por Kaneko, Harvey e Bruss ${ }^{11}$ e Birgel Junior et al. ${ }^{18}$. Foi encontrada linfocitose quando comparado aos valores encontrados por Aengwanich, Chantiratikul e Pamok ${ }^{12}$ e Oliver et al. ${ }^{13}$, porém os autores Kaneko, Harvey e Bruss ${ }^{11}$ e Birgel Junior et al. ${ }^{18}$ sugerem como normais valores semelhantes aos encontrados. Os valores observados para eosinófilos estão dentro dos intervalos sugeridos como normais por Kaneko, Harvey e Bruss $^{11}$; Aengwanich, Chantiratikul e Pamok ${ }^{12}$; Oliver et al. ${ }^{13}$ e Birgel Junior et al. ${ }^{18}$.

Os bovinos confinados são submetidos a muitas situações adversas, tais como aglomerações dos animais que levam ao estresse, agressão respiratória por poeira, 
e possibilidade de desenvolver coccidiose pela condição ambiental ${ }^{19}$. Essas situações adversas fazem com que o metabolismo animal se modifique para manter suas funções fisiológicas em completa homeostase, se estas forem superiores a capacidade do organismo em se manter em equilíbrio, as doenças da produção podem ser evidenciadas, causando prejuízos à saúde animal e prejuízos econômicos aos produtores.

A leucocitose fisiológica, devido à ação da adrenalina, provoca uma neutrofilia transitória e/ou linfocitose, e raramente ocorre aumento no número de monócitos e eosinófilos ${ }^{15}$. Aneutrofilia pode ser acompanhada por linfocitose e os outros tipos leucocitários permanecem dentro do intervalo de referência no sangue periférico, resultados estes, semelhantes aos encontrados no presente trabalho ${ }^{16}$.

A leucocitose fisiológica induzida pela adrenalina, em bovinos, é caracterizada por neutrofilia com aumento do número de linfócitos e monócitos e moderada eosinopenia ${ }^{5}$. No presente estudo foi encontrado leucocitose por neutrofilia e linfocitose, o que sugere estresse dos animais, provavelmente no momento da contenção e da punção venosa. A alta ingestão de concentrado adicionado de glicerina pode gerar transtornos metabólicos que exigem a busca da homeostase do organismo, alterando o quadro hematológico dos animais.

Os valores da contagem global de leucócitos e a contagem diferencial (neutrófilos segmentados e bastonetes, monócitos, linfócitos, basófilos e eosinófilos) encontrados são mostrados na tabela 3 .

A alta ingestão de concentrado com adição de glicerina na dieta gera aumento do metabolismo hepático, devido à maior ação do metabolismo energético do animal. As lesões hepáticas devidas à infiltração de gordura derivada da lipomobilização aumentam a possibilidade de sofrer transtornos como cetose, hipocalcemia e deslocamento de abomaso ${ }^{21}$, que causariam prejuízos ao desempenho dos animais, podendo levá-los ao óbito. Estas afecções podem gerar um processo inflamatório, atraindo células de defesa (polimorfonucleares e mononucleares) à corrente sanguínea.

Além dos efeitos sobre a contagem de leucócitos totais e neutrófilos segmentados, evidenciou-se efeito linear crescente sobre as concentrações de monócitos, que pode ser explicado devido à maior ação de órgãos hematopoiéticos, como o fígado, porém não suficiente para que ocorresse uma monocitose.

Apesar de evidenciar leucocitose por neutrofilia nos animais acompanhada de linfocitose, estes não

Tabela 3 - Concentrações de leucócitos totais, neutrófilos segmentados e bastonetes, monócitos, linfócitos, basófilos e eosinófilos

\begin{tabular}{|c|c|c|c|c|c|c|c|c|c|c|c|}
\hline \multirow{2}{*}{ Item $^{\mathrm{m}}$} & \multicolumn{5}{|c|}{ Glicerina bruta, (\%) } & \multirow{2}{*}{ Média } & \multicolumn{5}{|c|}{ Contrastes } \\
\hline & 0 & 7,5 & 15 & 22,5 & 30 & & $\mathrm{~L}^{\mathrm{h}}$ & $Q^{i}$ & $\mathrm{C}$ & $0 v s$. gli $^{\mathrm{k}}$ & $\mathrm{CV}^{1}(\%)$ \\
\hline $\mathrm{LT}^{\mathrm{a}}$ & 12,34 & 11,73 & 12,62 & 13,43 & 11,12 & 12,25 & NS & NS & $* *$ & NS & 21,40 \\
\hline $\mathrm{NS}^{\mathrm{b}}$ & 3,15 & 2,83 & 3,26 & 4,28 & 3,01 & 3,31 & NS & NS & $* *$ & NS & 48,32 \\
\hline $\mathrm{NB}^{\mathrm{c}}$ & 0,08 & 0,02 & 0,05 & 0,04 & 0,05 & 0,05 & NS & NS & NS & NS & 191,53 \\
\hline $\mathrm{MON}^{\mathrm{d}}$ & 0,02 & 0,04 & 0,05 & 0,07 & 0,07 & 0,05 & * & NS & NS & NS & 58,35 \\
\hline LINFe $^{e}$ & 8,70 & 8,49 & 8,71 & 8,72 & 7,55 & 8,43 & NS & NS & NS & NS & 26,68 \\
\hline $\mathrm{BAS}^{\mathrm{f}}$ & 0,00 & 0,00 & 0,00 & 0,00 & 0,00 & 0,00 & - & - & - & - & - \\
\hline $\mathrm{EOS}^{\mathrm{g}}$ & 0,20 & 0,33 & 0,44 & 0,33 & 0,47 & 0,36 & NS & NS & NS & NS & 140,43 \\
\hline
\end{tabular}

${ }^{\mathrm{a}}$ Leucócitos totais; ${ }^{\mathrm{b}}$ Neutrófilos segmentados; ${ }^{\mathrm{C}}$ Neutrófilos bastonetes; ${ }^{\mathrm{d}}$ Monócitos; ${ }^{\mathrm{e} L i n f o ́ c i t o s ; ~}{ }^{\mathrm{f}}$ Basófilos; ${ }^{\mathrm{g}}$ Eosinófilos; ${ }^{\mathrm{h}}$ Linear; ${ }^{\mathrm{i}}$ Quadrático; ${ }^{j}$ Cúbico; ${ }^{\mathrm{k}}$ Controle versus os outros tratamentos; ${ }^{1}$ Coeficiente de variação; ${ }^{\mathrm{m}} \mathrm{X} 10^{3} / \mu \mathrm{L} ;{ }^{*}<0,05 ;{ }^{* *}<0,01$; NS $=$ Não significativo 
apresentaram sinais clínicos durante o período experimental. Assim, pode-se inferir que as dietas podem ter proporcionado alterações metabólicas, porém não suficientes para causar prejuízos à saúde e ao desempenho dos animais confinados.

\section{Conclusão}

A inclusão da glicerina bruta em dietas de bovinos de corte confinados promove alterações no quadro eritrocitário e leucocitário destes animais. Mais estudos são necessários para avaliar a influência da glicerina sobre o quadro hematológico em um perío-

\section{Referências}

1. LOPES, M. A.; MAGALHÃES, G. P. Análise de rentabilidade de terminação de bovinos de corte em confinamento: um estudo de caso. Arquivo Brasileiro de Medicina Veterinária e Zootecnia, v. 57, n. 3, p. 374-379, 2005.

2. RIO DE JANEIRO. (Estado). Ministério de Minas e Energia. MME. Biocombustíveis: biodiesel obrigatório. Brasilia, 2010. Disponível em: <http://www.anp.gov.br>. Acesso em: 10 Jan. 2010.

3. FERRARI, R. A.; OLIVEIRA, V. S.; SCABIO, A. Biodiesel de soja: taxa de conversão em ésteres etílicos, caracterização físico-química e consumo em gerador de energia. Química Nova, v. 28, n. 1, p. 19-23, 2005.

4. MOTA, C. J. A.; SILVA, C. X. A.; GONÇALVES, V.L.C. Gliceroquímica: Novos produtos e processos a partir da glicerina de produção de biodiesel. Química Nova, v. 32, n. 3, p. 639-648, 2009.

5. SILVA, G. A.; SOUZA, B. B. de; ALFARO, C. E. P.; AZEVEDI NETO, J.; AZEVEDO, S. A.; SILVA, E. M. N. da.; SILVA, R. M. $\mathrm{N}$. da. Influência da dieta com diferentes níveis de lipídeo e proteína na resposta fisiológica e hematológica de reprodutores caprinos sob estresse térmico. Ciência e Agrotecnologia, v. 30, n. 1, p. 154-161, 2006.

6. SILVA, D. G.; SILVA, P. R. L.; FAGLIARI, J. J. Hemograma e perfil bioquímico sérico, inclusive hemogasométrico, de bezerros infectados experimentalmente com Salmonella Dublin. Arquivo Brasileiro de Medicina Veterinária e Zootecnia, v. 62, n. 2, p. 251-257, 2010.

7. PEIXOTO, A. P. C.; COSTA, J. N.; KOHAYAGAWA, A.; TAKAHIRA, R. K.; SAITO, M. E. Hemograma e metabolismo oxidativo dos neutrófilos de bovinos da raça Holandesa preta e branca - Influência dos fatores etários. Revista Brasileira de Saúde e Produção Animal, v. 3 n. 1, p. 16-20, 2002.

8. OlIVEIRA, J. N. F.; MELO, M. M.; LAGO, L. A.; NASCIMENTO, E. F. Hemograma, bioquímica sérica e histologia da biópsia hepática de bovinos após administração de polpa cítrica. Arquivo Brasileiro e Medicina Veterinária e Zootecnia, v. 57, n. 3, p. 418-422, 2005.

9. GONZÁLEZ, F. H. D.; SCHEFFER, J. F. S. Perfil sangüíneo: ferramenta de análise clínica, metabólica e nutricional. In: I SIMPÓSIO DE PATOLOGIA CLÍNICA VETERINÁRIA DA do maior de confinamento, com o objetivo de evitar perdas no desempenho animal. O estudo de parâmetros avaliativos presentes no hemograma de bovinos alimentados com diferentes doses de glicerina não mostrou alterações que indicassem prejuízos à saúde dos animais.

\section{Agradecimento}

Os autores agradecem à FAPESP pelo financiamento do projeto e concessão de bolsas de estudo e à Caramuru Alimentos S.A. pelo fornecimento de parte dos ingredientes utilizados neste estudo.

REGIÃO SUL DO BRASIL, 2003, Porto Alegre. Anais... Porto Alegre: UFRGS, 2003. p. 73-87.

10.PAYNE, J. M.; PAYNE, S. The metabolic profile test. Oxford University Press. New York, 1987.

11.KANEKO, J.J.; HARVEY, J.W.; BRUSS, M.C. Clinical biochemistry of domestic animals. 6.ed. San Diego: Academic Press, 2008. 928 p.

12.AENGWANICH, W.; CHANTIRATIKUL, A.; PAMOK, S. Effect of Seasonal Variations on Hematological Values and Health Monitor of Crossbred Beef Cattle at Slaughterhouse in Northeastern Part of Thailand. American-Eurasian Journal of Agricultural \& Environmental Sciences, v. 5, n. 5, p. 644648, 2009.

13. OLIVER, J. W.; SCHULTZE, A. E.; ROHRBACH, B. W.; FRIBOURG, H.; INGLE, T.; WALLER, J. C. Alterations in hemograms and serum biochemical analytes of steers after prolonged consumption of endophyte-infected tall fescue. Journal of Animal Science, v. 78, n. 4, p. 1029-1035, 2000.

14.LEE, J. A.; ROUSSEL, J. D.; BEATTY, J. F. Effect of temperature season on bovine adrenal cortical function, blood cell profile, and milk production. Journal of Dairy Science, v. 59, n. 1, p. 104-108, 1974.

15. JAIN, N. C. Essentials of veterinary hematology. Philadelphia: Lea \& Febiger, 1993. 417 p.

16. FELDMAN, B. F.; ZINKL, J. G.; JAIN, N. C. Schalm's veterinary hematology. 5 . ed. Philadelphia: Lippincott Willians \& Wilkins. 2000. 1174 p.

17. KEER, G. M. Exames laboratoriais em medicina veterinária: Bioquímica clinica e hematologia. 2. ed. São Paulo: Roca, 2003. 436 p.

18. BIRGEL JUNIOR, E. H; D'ANGELINO, J. L.; BENESI, F. J.; BIRGEL, E. H. Reference values of the leucogram of the Jersey cattle, raised in São Paulo State. Brazilian Journal of Veterinary Research and Animal Science, v. 38, n. 3, p. 136$141,2001$.

19.DUTRA, I. S. Programa sanitário para confinamentos. In: SEMINÁRIO INTERNACIONAL NUTRON SOBRE NUTRIÇÃO DE BOVINOS, 3., 2001, Goiânia: entidade. Anais... Goiânia, 2001. p. 3. 
20.THAYLOR, J. A. Leukocyte responses in ruminants. In: FELDMAN, B. F.; ZINKL, J. G.; JAIN, N. C. Schalm's veterinary hematology. 5. ed. Philadelphia: Lippincott Willians \& Wilkins, 2001. p. 174
21.AMETAJ, B. N. A new understanding of the causes of fatty liver in dairy cows. Advances in Dairy Technology, v. 17, p. 97-112, 2005. 